\title{
Chemical Recycling of Poly(bisphenol A carbonate) by Glycolysis under 1,8-Diazabicyclo[5.4.0]undec-7-ene Catalysis
}

\author{
Eugenio Quaranta, *(1) Clara Castiglione Minischetti, and Giuseppe Tartaro \\ Dipartimento di Chimica, Università degli Studi “Aldo Moro” di Bari, Campus Universitario, Via E.Orabona, 4, 70126 Bari, Italy \\ Supporting Information
}

ABSTRACT: The glycolysis reaction of poly(bisphenol A carbonate) (PC) has been explored under 1,8diazabicyclo[5.4.0] undec-7-ene (DBU) catalysis as a potential route to valorize $\mathrm{PC}$ wastes by chemical recycling. The amidine base is an active catalyst of PC glycolysis and, under suitable conditions, promotes effectively and selectively the depolymerization of the polymeric material with 1,2-propanediol or glycerol to give the monomer bisphenol A (BPA) and the relevant cyclic carbonate. The depolymerization process has been investigated under solventless conditions, using diol/ triol as the reagent and reaction medium, and also in an auxiliary solvent such as tetrahydrofuran (THF) that is able to dissolve the polymer. The influence of a few experimental parameters (temperature, catalyst load, and reaction time) on the selectivity to cyclic carbonate has been studied. High selectivity to cyclic carbonate has been attained by carrying out the depolymerization reaction in THF and using mild temperature conditions and a stoichiometric amount of polyol. The catalyst can be recovered from the reaction mixture as a $\mathrm{BPA} / \mathrm{DBU}$ adduct and effectively recycled in a successive run.

\section{INTRODUCTION}

Chemical recycling of waste plastics is a useful strategy to reduce the environmental and social impact of this typology of wastes. This approach, which regards the waste as a potential resource rather than a mere refuse, implies the chemical conversion of waste polymers into valuable chemicals and provides not only a fascinating alternative to more conventional technological solutions (landfill, mechanical recycling, and energy recovery) but also a smart response to the current worldwide need of recycling carbon and saving energy. ${ }^{1,2}$

Poly(bisphenol A carbonate) (PC; 1) is one of the most widely used thermoplastics whose market is in rapid expansion. The rise in the utilization of PC calls for the development of after-use treatments. Chemical recycling may be a suitable way of valorizing waste PC., In recent years, besides pyrolytic approaches, ${ }^{3,4}$ great attention has been paid to those protocols implying the cleavage of carbonate bond by hydrolysis, ${ }^{3-6}$ aminolysis, ${ }^{7}$ and alcoholysis. ${ }^{3,4,8-10}$ These methods not only provide a potential route to the regeneration of the starting monomer bisphenol A (BPA; 2), which can be reused to produce new virgin $\mathrm{PC}$, but can also allow the synthesis of chemicals with the added value. ${ }^{3,4}$ In this paper, we have focused on the glycolysis reaction (eq 1; Scheme 1). This reaction is a potential route to recover BPA, and it also provides a synthetic entry into cyclic carbonates, a class of compounds widely used as solvents or intermediates in chemical synthesis. In principle, also the cyclic carbonate can be reused, together with recovered $\mathrm{BPA}$, to regenerate virgin
Scheme 1. PC Glycolysis (Eq 1)

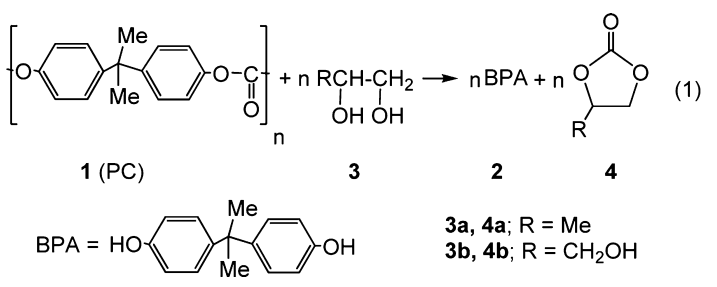

PC. In fact, cyclic carbonates, for a long time prepared from toxic phosgene or $\mathrm{CO}$ as sources of the carbonyl group, nowadays can be more safely obtained by the cycloaddition of $\mathrm{CO}_{2}$ to epoxides: ${ }^{11}$ this reaction is currently exploited at an industrial scale for the synthesis of dimethyl carbonate, used as a co-monomer (together with BPA) in the manufacture of PC. ${ }^{12}$

Han et al. studied the glycolysis of PC in ethylene glycol (EG) in the absence of any catalyst. ${ }^{13}$ At $453 \mathrm{~K}$, depolymerization proceeded slowly with low BPA yield $(30 \%$, after $6 \mathrm{~h})$. At $493 \mathrm{~K}$, the conversion of the polymer into the monomer was practically quantitative in $85 \mathrm{~min}$, but, under the working conditions, the coproduced cyclic carbonate decomposed fast with the evolution of $\mathrm{CO}_{2}$.

Received: May 24, 2018

Accepted: June 18, 2018

Published: July 3, 2018 
Inorganic bases, such as $\mathrm{NaOH}^{14}$ or also $\mathrm{Na}_{2} \mathrm{CO}_{3}{ }^{15}$ turned out to be active catalysts of PC glycolysis and, at $453 \mathrm{~K}$, under solvolytic conditions, promoted the depolymerization of $\mathrm{PC}$ in $\mathrm{EG}^{14,15}$ or 1,2-propanediol (1,2-PD; $\left.3 \mathrm{a}\right)^{15}$ to give a mixture of 2 and BPA mono- and bis-(hydroxyalkyl)-ethers. In these processes, the cyclic carbonate $\mathbf{4}$ formed as transient species as the used catalysts, under the working conditions, promoted fast not only the decomposition of 4 by decarboxylation ${ }^{14}$ but also the reaction of 4 with 2 to give BPA mono- (5) and bishydroxyalkylation (6) products (Scheme 2). ${ }^{14,15}$

Scheme 2. Hydroxyalkylation of BPA (2) with Propylene Carbonate (4a): Formation of Mono-Hydroxypropyl-BPA (MHP-BPA) and Bis-Hydroxypropyl-BPA (BHP-BPA) Derivatives

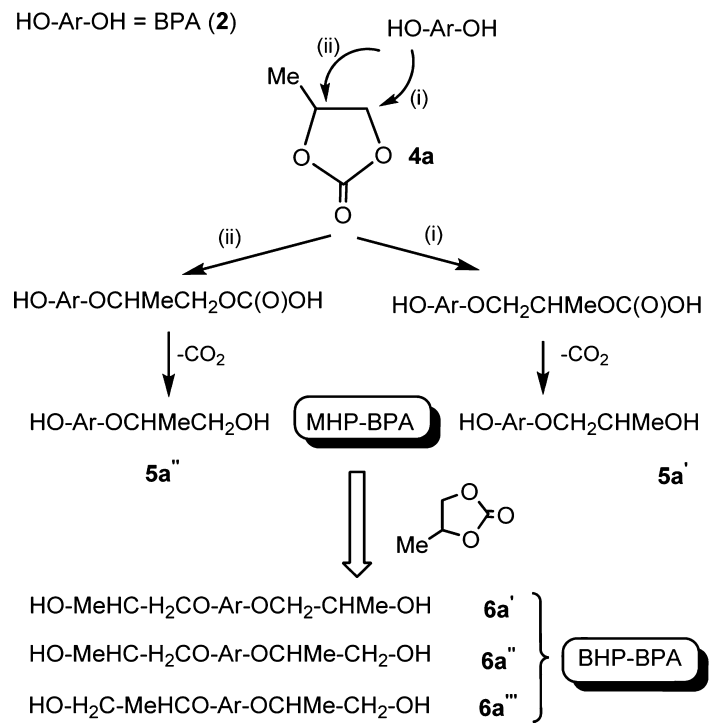

The utilization of nonconventional heating techniques such as microwave irradiation did not improve the selectivity to cyclic carbonate. ${ }^{16,17}$ The success of reaction 1 depends (a) on the choice of a suitable catalyst that, although being effective in catalyzing carbonate formation (eq 1), should exhibit a poor tendency to promote both carbonate decomposition and BPA hydroxyalkylation (Scheme 2) and also requires (b) the careful tuning of right working conditions. To date, the isolation of cyclic carbonate from reaction 1 has been described only in rare cases. Oku, using $\mathrm{NaOH}$ or $\mathrm{KOH}$ as catalysts, first reported the synthesis of $\mathrm{BPA}$ and glycerol carbonate (4b) from the reaction of $\mathrm{PC}$ and a stoichiometric amount of glycerol (3b), at $373 \mathrm{~K}$, in dioxane as the auxiliary solvent. ${ }^{18}$ Alkali bases are relatively cheap catalysts, but they cannot be recycled, are wasted at the end of the process, may cause equipment corrosion, and generate environmental problems. Recently, a more complex recyclable catalytic system based on an ionic liquid $\left(\mathrm{Bu}_{4} \mathrm{NCl}\right)$ and $\mathrm{ZnO}$ nanoparticles was found to be effective in promoting, at $373 \mathrm{~K}$ in tetrahydrofuran (THF), the depolymerization of PC with diols (1,2-PD; 1,3butanediol) or glycerol to give the relevant cyclic carbonates besides 2. However, the process required relatively long conversion times $(7-14 \mathrm{~h})$ as well as a strong excess (vs PC) of the used polyol. ${ }^{19}$ To date, the search for effective reusable catalytic systems able to promote reaction 1 selectively under conditions more appealing from a practical point of view is still a challenging task. In this work, we have focused on an organocatalyst such as 1,8-diazabicyclo[5.4.0]undec-7-ene (DBU). Elsewhere, we have shown that DBU can promote the carbonylation of nucleophiles $\mathrm{NuH}$ with carbonic acid diesters (Scheme 3, eq 2): ${ }^{10,20-22}$ DBU can work both as a

Scheme 3. DBU-Promoted Carbonylation of Nucleophiles $\mathrm{NuH}$ with Carbonic Acid Diesters (RO) ${ }_{2} \mathrm{CO}$

$$
\mathrm{NuH}+(\mathrm{RO})_{2} \mathrm{CO} \stackrel{\mathrm{DBU}}{\rightleftarrows} \mathrm{NuCO}_{2} \mathrm{R}+\mathrm{ROH} \quad(2)
$$
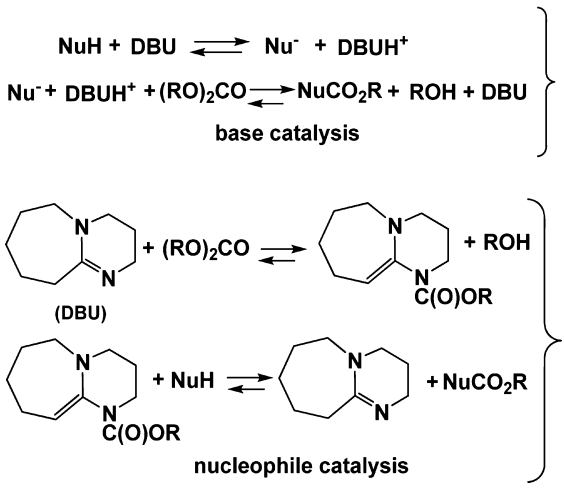

base, activating $\mathrm{NuH}$ through the formation of the more nucleophilic anion $\mathrm{Nu}^{-}$(Scheme 3; base catalysis), ${ }^{22}$ and as a nucleophile capable of activating the carbonate group through the formation of a $\mathrm{N}$-carbonyl-substituted ketene aminal (Scheme 3; nucleophilic catalysis), ${ }^{20-22}$ which can effectively transfer the $\mathrm{CO}_{2} \mathrm{R}$ group to nucleophiles $\mathrm{NuH}$ such as $\mathrm{N}$ heteroaromatics (pyrrole) and even alcohols. ${ }^{20}$ This reactivity has been recently exploited in a very recent study, wherein we have shown that DBU is an effective selective catalyst of PC alcoholysis. ${ }^{10}$ Herein, we report on the activity of DBU as the catalyst of reaction 1 and describe a few novel simple efficient protocols for the glycolysis and glycerolysis of PC using the amidine superbase as the recyclable catalyst.

\section{EXPERIMENTAL SECTION}

2.1. General Methods and Materials. In this study, pure PC pellets ( $3 \mathrm{~mm}$ length $\times 2 \mathrm{~mm}$ diameter) were used as a model of waste PC. The used polycarbonate may contain or not a chain terminator. Polycarbonate used in the experiments with 1,2-PD was from Aldrich $\left(M_{\mathrm{w}} \approx 64000\right.$ by gel permeation chromatography). Scheme $S 1$ shows a sketch of the polymeric chain and highlights the nature of the additive, 4-cumylphenol (4-CP), used as a chain terminator. ${ }^{23}$ The spectroscopic characterization [NMR and Fourier-transform infrared (FTIR)] of this polymeric material has been reported elsewhere. ${ }^{6}$ The molar ratio of 4-CP/BPA in the used polymer was equal to 0.035 , as determined by $\mathrm{NMR}^{6}$ Accordingly, the moles of BPA units $\left(n_{\mathrm{BPA}}{ }^{\circ}\right)$ and the moles of $\mathrm{CO}_{3}$ groups $\left(n_{\mathrm{CO}_{3}}\right)$ incorporated in $w(g)$ of PC feed have been calculated by means of eqs $\mathrm{S} 1$ and $\mathrm{S} 2$, respectively.

Polycarbonate used in the glycerolysis experiments was from SABIC $\left(M_{\mathrm{w}} \approx 40000\right.$ by GPC). Figures S1-S3 show, respectively, the ${ }^{1} \mathrm{H}$ and ${ }^{13} \mathrm{C}$ NMR and attenuated total reflection (ATR)-FTIR spectra of the polymer. In this case, the moles of BPA $\left(n_{\mathrm{BPA}}{ }^{\circ}\right)$ and $\mathrm{CO}_{3}$ groups $\left(n_{\mathrm{CO}_{3}}\right)$ present in $w(g)$ of feed PC have been calculated according to eq S3.

Yields of 2 and 4 were calculated through eqs 3 and 4, respectively, 


$$
\begin{aligned}
& \text { yield of } 2(\%)=\left(n_{\mathrm{BPA}} / n_{\mathrm{BPA}}{ }^{\circ}\right) \times 100 \\
& \text { yield of } 4(\%)=\left(n_{\text {carbonate }} / n_{\mathrm{CO}_{3}}\right) \times 100
\end{aligned}
$$

where $n_{\mathrm{BPA}}$ and $n_{\text {carbonate }}$ were, respectively, the moles of $\mathbf{2}$ and 4 determined by $\mathrm{GC}$ or isolated.

1,2-PD (3a) and glycerol (3b) were Fluka and Aldrich products, respectively. THF and diethyl ether were previously dried according to conventional methods $\left(\mathrm{P}_{2} \mathrm{O}_{5} ; \mathrm{Na} /\right.$ benzophenone $)^{24}$ and stored under $\mathrm{N}_{2}$. DBU (Aldrich) was used as received and manipulated under an inert gas atmosphere to prevent any contamination from atmospheric $\mathrm{CO}_{2}$ or moisture.

GC analyses were performed with a HP 5890 Series II or a THERMO Scientific TRACE 1310 gas-chromatograph (capillary column: Heliflex AT-5, $30 \mathrm{~m} \times 0.25 \mathrm{~mm}, 0.25 \mu \mathrm{m}$ film thickness). GC-(mass spectrometry) MS analyses were carried out with Shimadzu GC-17A linked to a Shimadzu GC-MS QP5050 selective mass detector (capillary column: Supelco MDN-5S, $30 \mathrm{~m} \times 0.25 \mathrm{~mm}, 0.25 \mu \mathrm{m}$ film thickness). IR spectra were recorded on a Shimadzu FTIR Prestige 21 spectrophotometer or a PerkinElmer Frontier MIR/FIR spectrophotometer equipped with a Pike GladiATR (diamond crystal) accessory. NMR spectra were recorded with a Varian Inova 400 spectrometer or with an Agilent 500 instrument. Chemical shifts are in $\delta(\mathrm{ppm})$ versus tetramethylsilane.

2.2. PC Glycolysis with 1,2-PD under Solvolytic Conditions: General Procedure. The glycolysis reaction was carried out in a $\sim 40 \mathrm{~mL}$ Schlenk tube equipped with a Sovirel screw cap and Torion stopcock. The polycarbonate was suspended in the diol under an inert gas $\left(\mathrm{N}_{2}\right)$ stream, and the catalyst was added. The reactor, once charged with the reactants, was sealed and dipped into an electrically heated silicon oil bath, and the suspension was stirred at the working temperature until complete depolymerization of PC (disappearance of the PC pellets). The reaction mixture was cooled to room temperature, diluted with THF, and analyzed by FTIR, GC-MS, and GC using $n$-dodecane as the internal standard.

In a few experiments, heating was prolonged until the full consumption of propylene carbonate (4a) initially formed (see, later, Table 4). BPA, as well as MHP-BPA and BHP-BPA species formed by the reaction of 2 with $\mathbf{4 a}$ (Scheme 2), was separated by chromatography as reported hereafter. The reaction mixture, cooled to room temperature, was treated with distilled $\mathrm{H}_{2} \mathrm{O}$ and then extracted with diethyl ether. The organic phase was dried over $\mathrm{MgSO}_{4}$ and fractionated on a silica gel column using, as eluent, petroleum ether/diethyl ether 1:1 (v/v) until elution of 4-CP and BPA and then petroleum ether/diethyl ether $1: 2(\mathrm{v} / \mathrm{v})$ until elution of the mixture of MHP-BPA derivatives (5a' and $\left.\mathbf{5 a}^{\prime \prime}\right)$. Afterward, the composition of the mobile phase was progressively enriched in diethyl ether [petroleum ether/diethyl ether: $1: 3(\mathrm{v} / \mathrm{v}) \rightarrow 1: 5$ $(\mathrm{v} / \mathrm{v}) \rightarrow 0: 1(\mathrm{v} / \mathrm{v})]$ until elution of the mixture of BHP-BPA isomers $\left(6 a^{\prime}-6 a^{\prime \prime \prime}\right)$. The NMR characterization of the mixture of MHP-BPA derivatives (5a) as well as the mixture of BHPBPA isomers (6a) has been reported in the Supporting Information (Figures S4-S7).

2.3. PC Glycolysis with 1,2-PD in THF: General Procedure. The glycolysis reaction was carried out in a suitable glass reactor analogous to that described above. The reactor, once charged with the reactants and the catalyst, was sealed, and the reaction mixture, containing also $n$-dodecane (internal standard) if used, was stirred at the working temperature. The progress of the reaction was monitored by measuring the formation of $\mathbf{4 a}$ over time by GC. The complete conversion of the polymer was further confirmed spectroscopically by observing the disappearance of the FTIR absorption of the polycarbonate at $1778 \mathrm{~cm}^{-1}$. The products can be separated by column chromatography. Herein, we report the details of one of these experiments. To a THF $(5 \mathrm{~mL})$ solution of PC $(0.512 \mathrm{~g}), 1,2-\mathrm{PD}(150 \mu \mathrm{L})$ and DBU $(30 \mu \mathrm{L} ; 10 \mathrm{~mol} \%$ vs $\left.n_{\mathrm{CO}_{3}}\right)$ were added. The reaction mixture was reacted at 373 $\mathrm{K}$ for $2.5 \mathrm{~h}$ and, then, analyzed by GC ( $4 \mathrm{a}$ yield: $\geq 99 \%$ ). Both 4a and 2 were separated with high yield (91 and 94\%, respectively) on a silica gel column using, as eluents, $\mathrm{CHCl}_{3}$ and, after elution of $\mathbf{4 a}$, diethyl ether.

2.3.1. Catalyst Recovery and Recycling. The catalyst can be recovered through a procedure analogous to that described in ref 10. At the end of the catalytic run, the solvent (THF) was evaporated in vacuum. The residue was washed with diethyl ether. The ethereal phase, after addition of $n$-dodecane, was analyzed by GC. The material insoluble in diethyl ether was dried in vacuum and characterized by ${ }^{1} \mathrm{H}$ NMR (Figure S8) as a BPA/DBU adduct. ${ }^{10}$ In accordance with the outcomes of our previous studies on DBU-promoted PC alcoholysis, ${ }^{10}$ the $\mathrm{BPA} / \mathrm{DBU}$ ratio was found to be equal to $2.5: 1 \mathrm{~mol} / \mathrm{mol}$ as established by means of the ${ }^{1} \mathrm{H}$ integral spectrum. The recovered adduct, once dissolved in THF and after addition of fresh PC and the diol, was reusable in a new run.

2.4. PC Glycerolysis under Solvolytic Conditions: General Procedure. The experimental apparatus was similar to that described for solventless glycolysis experiments (see 2.2). To the suspension of $\mathrm{PC}$ in the triol, the catalyst was added under a $\mathrm{N}_{2}$ stream. The glass reactor was sealed, and the reaction mixture was stirred at the working temperature until complete PC depolymerization (disappearance of the PC pellets). The reaction mixture was cooled to ambient temperature, analyzed qualitatively by FTIR and GC-MS, treated with $\mathrm{H}_{2} \mathrm{O}$, and extracted several times with diethyl ether. The resulting ethereal solution was dried over $\mathrm{MgSO}_{4}$ and, after addition of $n$-dodecane (internal standard), analyzed by GC for the quantitation of 2. BPA was also isolated by chromatography on a silica gel column using, as eluent, a petroleum ether/ethyl acetate gradient [from 6:1 to $2: 1(\mathrm{v} / \mathrm{v})$ ].

2.5. PC Glycerolysis in THF: General Procedure. Into a suitable glass reactor (see 2.3), containing the THF solution of the polymer, glycerol, the catalyst, and $n$-hexadecane (internal standard), if used, were introduced under a $\mathrm{N}_{2}$ stream. The glass reactor was sealed, and the reaction mixture was stirred at the working temperature. The progress of the reaction was monitored by measuring the formation of $4 \mathbf{b}$ over time by GC. The quantitative conversion of the polymer was further confirmed spectroscopically by observing the disappearance of the FTIR absorption of the polycarbonate at $1778 \mathrm{~cm}^{-1}$. The products can be separated by column chromatography. Herein, we report the details of one of these experiments. To the THF $(5 \mathrm{~mL})$ solution of PC $(0.517 \mathrm{~g})$, glycerol $(150 \mu \mathrm{L})$ and $\mathrm{DBU}\left(15 \mu \mathrm{L} ; 5 \mathrm{~mol} \%\right.$ vs $\left.n_{\mathrm{CO}_{3}}\right)$ were added. The reaction mixture was reacted at $333 \mathrm{~K}$ for $3 \mathrm{~h}$ and cooled to room temperature. After addition of $\mathrm{CH}_{3} \mathrm{COOH}(6 \mu \mathrm{L})$, the solution was evaporated in vacuum, and the residue was fractionated on a silica gel column, first with petroleum ether/ ethyl acetate 9:1 (v/v), afterward with a mobile phase progressively enriched in ethyl acetate until elution of BPA 
(94\% yield), and, finally, with only ethyl acetate until elution of 4b ( $90 \%$ yield).

\section{RESULTS AND DISCUSSION}

3.1. DBU-Promoted Glycolysis of PC with 1,2-PD. In this work, 1,2-PD (3a) was selected as the reference diol. The glycolysis reaction was preliminarily investigated in the absence of any catalyst, under solvolytic conditions, using diol as the reactant and reaction medium (Table 1). At $453 \mathrm{~K}$ (1,2-PD/

Table 1. PC $(\sim 0.200 \mathrm{~g})$ Depolymerization in 1,2-PD $(2 \mathrm{~mL}$; $\left.1,2-\mathrm{PD} / \mathrm{PC} \approx 12 \mathrm{~m} / \mathrm{m} ; \mathrm{mol}_{1,2-\mathrm{PD}} / \mathrm{mol}_{\mathrm{CO}_{3}} \approx 3.6\right)$, at $453 \mathrm{~K}$, in the Absence of Any Catalyst

\begin{tabular}{clcc} 
entry & \multicolumn{1}{c}{$t^{a}(\mathrm{~h})$} & $\mathbf{4 a}^{b}(\%)$ & $\mathrm{BPA}^{b}(\%)$ \\
1 & $1.83(1.83)$ & 94 & 95 \\
2 & $28(2)$ & 78 & 81
\end{tabular}

${ }^{a}$ Reaction time. The value in parentheses is the time after which the depolymerization of PC was quantitative (disappearance of the PC pellets). ${ }^{b} \mathrm{GC}$ yield.

$\mathrm{PC} \approx 12 \mathrm{~m} / \mathrm{m}$ ), the depolymerization of 1 proceeded heterogeneously because the polymer was poorly soluble in 3a under the working conditions. The reaction was stopped after $\sim 2$ h (entry 1, Table 1), when depolymerization was quantitative (i.e., disappearance of the polymer pellets) and a homogeneous system was obtained. Both 2 and $\mathbf{4 a}$ were obtained in high yield (95 and 94\%, respectively). However, prolonging the reaction time caused a sensible diminution of both 2 and 4 a yield ( 81 and 78\%, respectively; entry 2, Table 1) because of side formation of MHP-BPA and BHP-BPA derivatives, as well as other species, identified by GC-MS, such as 1-[4-(2-phenylpropan-2-yl)phenoxy]propan-2-ol (the 2-hydroxypropylether of 4-CP; $270 \mathrm{~m} / z$ ) and $\mathrm{PhOH}$, 4isopropenylphenol (4-IPP) and 4-isopropylphenol (4-IPPH), which are products of thermal decomposition of BPA. ${ }^{25}$

$\mathrm{DBU}$ is an active catalyst of PC glycolysis in 1,2-PD. In the presence of the amidine base, the depolymerization of 1 was markedly faster and, using a catalyst load as high as $10 \mathrm{~mol} \%$ $\left(\mathrm{mol}_{\mathrm{DBU}} / \mathrm{mol}_{\mathrm{CO}_{3}}\right)$, was complete in $5 \mathrm{~min}$ (Table 2 and Figure $1)$. The reacting system remained heterogeneous throughout the reaction time, that is, until complete disappearance of the

Table 2. Propylene Carbonate Yields and PC $(\sim 0.200 \mathrm{~g})$ Depolymerization Times in 1,2-PD $(2 \mathrm{~mL} ; 1,2-\mathrm{PD} / \mathrm{PC} \approx 12$ $\mathrm{m} / \mathrm{m} ; \mathrm{mol}_{1,2-\mathrm{PD}} / \mathrm{mol}_{\mathrm{CO}_{3}} \approx 3.6$ ), at $453 \mathrm{~K}$, in the Presence of DBU

$\begin{array}{cccccc}\text { entry } & \begin{array}{c}\mathrm{DBU} \\ (\mathrm{mL})\end{array} & \begin{array}{c}\mathrm{DBU}_{\text {molar }} \\ \operatorname{load}^{a}(\%)\end{array} & \begin{array}{c}\mathrm{DBU} \text { concentration } \\ \left(\mathrm{g}_{\mathrm{DBU}} / \mathrm{mL}_{1,2-\mathrm{PD}}\right)\end{array} & \begin{array}{c}t^{b} \\ (\mathrm{~min})\end{array} & \begin{array}{c}\text { 4a GC } \\ \text { yield }(\%)\end{array} \\ 1 & 0.012 & 10.4 & 0.006 & 5 & 84 \\ 2 & 0.006 & 5.1 & 0.003 & 8 & 85 \\ 3^{c} & 0.0012 & 1.0 & 0.0006 & 23 & 87 \\ 4 & & & & 110 & 94\end{array}$

${ }^{a}\left(\mathrm{~mol}_{\mathrm{DBU}} / \mathrm{mol}_{\mathrm{CO}_{3}}\right) \times 100 .{ }^{b}$ Reaction time: at that time, the depolymerization of PC was quantitative (100\%; disappearance of the PC pellets). ${ }^{c} \mathrm{BPA}$ was isolated with $82 \%$ yield: at the end of the catalytic run, the reaction mixture was partitioned between diethyl ether and distilled $\mathrm{H}_{2} \mathrm{O}$. The ethereal phase was dried over $\mathrm{MgSO}_{4}$ and fractionated on a silica gel column with petroleum ether/diethyl ether 1:1 (v/v) until elution of 4-CP (87\%) and, afterward, petroleum ether/diethyl ether 1:2 (v/v) until elution of BPA.

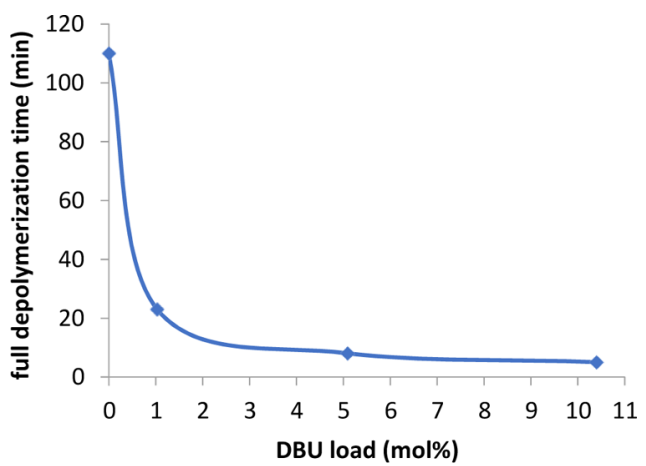

Figure 1. PC depolymerization time vs DBU molar load [ $\left(\mathrm{mol}_{\mathrm{DBU}} /\right.$ $\left.\mathrm{mol}_{\mathrm{CO}_{3}}\right) \times 100$ ]. Experimental conditions: PC, $\sim 0.200 \mathrm{~g} ; 1,2-\mathrm{PD}, 2$ $\mathrm{mL}\left(1,2-\mathrm{PD} / \mathrm{PC} \approx 12 \mathrm{~m} / \mathrm{m} ; \mathrm{mol}_{1,2-\mathrm{PD}} / \mathrm{mol}_{\mathrm{CO}_{3}} \approx 3.6\right) ; T, 453 \mathrm{~K}$.

PC pellets. The FTIR analysis of the reaction mixture (diluted in THF) showed the disappearance of the band of PC at 1778 $\mathrm{cm}^{-1}$ and the appearance of the new absorption at $1809 \mathrm{~cm}^{-1}$ assigned to 4a (Figure 2): the FTIR spectrum does not show

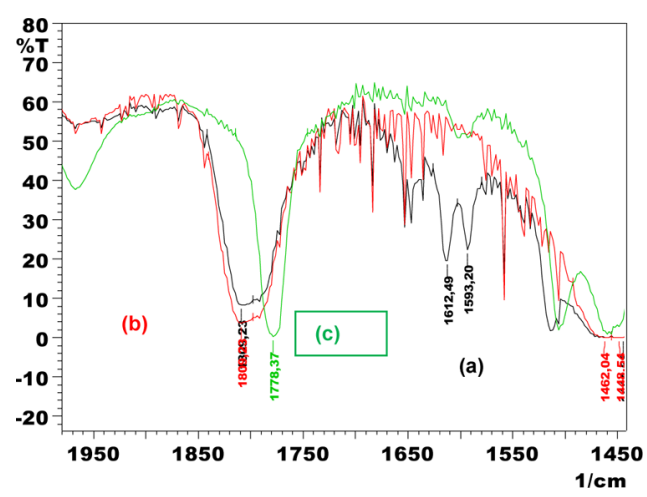

Figure 2. FTIR spectra. (a) Reaction mixture diluted in THF (2 mL) after disappearance of the PC pellets (100\% depolymerization); the bands at 1612 and $1593 \mathrm{~cm}^{-1}$ are due to the BPA product. (b) Propylene carbonate in $1,2-\mathrm{PD} / \mathrm{THF}(1: 1 \mathrm{v} / \mathrm{v})$. (c) PC in THF.

any other carbonyl absorption at lower wavenumbers excluding, thus, the presence in the reaction mixture of significant amounts of soluble oligomers or hydroxyalkyl-arylcarbonate species. This suggests that the latter species, once formed, react fast to give the final products.

The use of lower catalyst loads caused the lengthening of the depolymerization time. However, even by reducing the DBU load to $1 \mathrm{~mol} \%$, the increase of depolymerization time was contained within more than acceptable limits $(23 \mathrm{~min}$, at 453 $\mathrm{K})$. The selectivity to carbonate $4 \mathrm{a}$, measured when the depolymerization of $\mathbf{1}$ was complete, was lower than in the absence of the catalyst (in Table 2, entries 1-3 compared with entry 4), despite the fact that in the presence of DBU the reaction times were markedly shorter. This suggests that DBU may promote, in some degree, the further conversion of $4 \mathrm{a}$. However, the high selectivity observed ( $\geq 84 \%$; entries $1-3$, Table 2) indicates that $\mathbf{4 a}$ is quite stable under the working conditions and reacts with BPA only to a moderate extent to give MHP-BPA and BHP-BPA derivatives (Scheme 2).

As expected, the use of less severe reaction temperatures caused a significant slowdown of the depolymerization process (Table 3, Figure 3). The selectivity to $\mathbf{4 a}$, measured when PC depolymerization was quantitative, tends to decrease, albeit 
Table 3. Propylene Carbonate Yields and PC $(\sim 0.200 \mathrm{~g})$ Depolymerization Times in 1,2-PD $(2 \mathrm{~mL} ; 1,2-\mathrm{PD} / \mathrm{PC} \approx 12$ $\left.\mathrm{m} / \mathrm{m} ; \mathrm{mol}_{1,2-\mathrm{PD}} / \mathrm{mol}_{\mathrm{CO}_{3}} \approx 3.6\right)$ at Different Temperatures, in the Presence of $\sim 10 \mathrm{~mol} \% \mathrm{DBU}^{a}$

$\begin{array}{cccc}\text { entry } & T(\mathrm{~K}) & t^{b}(\min ) & \text { 4a GC yield }(\%) \\ 1 & 453 & 5 & 84 \\ 2 & 436 & 10 & 83 \\ 3 & 407 & 60 & 74 \\ 4 & 393 & 270 & 75\end{array}$

${ }^{a}\left(\mathrm{~mol}_{\mathrm{DBU}} / \mathrm{mol}_{\mathrm{CO}_{3}}\right) \times 100 .{ }^{b}$ Reaction time: at that time, the depolymerization of PC was quantitative (disappearance of the PC pellets).

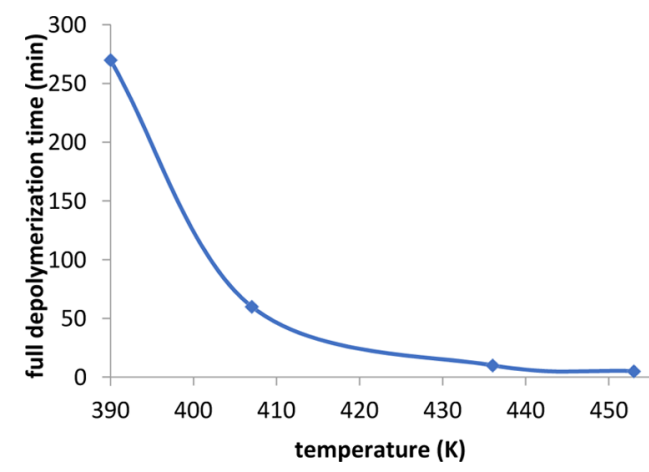

Figure 3. PC depolymerization time vs temperature. Experimental conditions: PC, $\sim 0.200 \mathrm{~g} ; 1,2-\mathrm{PD}, 2 \mathrm{~mL}(1,2-\mathrm{PD} / \mathrm{PC} \approx 12 \mathrm{~m} / \mathrm{m}$; $\left.\mathrm{mol}_{1,2-\mathrm{PD}} / \mathrm{mol}_{\mathrm{CO}_{3}} \approx 3.6\right)$; DBU molar load $\left[\left(\mathrm{mol}_{\mathrm{DBU}} / \mathrm{mol}_{\mathrm{CO}_{3}}\right) \times 100\right]$, $\sim 10 \mathrm{~mol} \%$.

slowly, on lowering temperature (Table 3). This effect was more evident at the lowest temperatures investigated at which the time required for the full depolymerization of $\mathbf{1}$ was markedly longer.

Prolonging heating after the complete depolymerization of 1 caused the quantitative conversion of $4 a$ within times that increased with decreasing either temperature or the catalyst load (Table 4). The disappearance of $\mathbf{4 a}$ was accompanied by the significant formation of MHP-BPA and BHP-BPA derivatives (Scheme 2), and minor amounts of other species, a few of which have been identified by GC-MS [PhOH (94 $m / z) ; 1,1^{\prime}$-oxydipropan-2-ol and 2-(2-hydroxypropoxy)-1propanol $(134 \mathrm{~m} / z)$; 4-IPPH $(136 \mathrm{~m} / z)$; 4-IPP $(134 \mathrm{~m} / z)$; 1-phenoxypropan-2-ol $(152 \mathrm{~m} / z)$; 1-[4-(2-phenylpropan-2yl)phenoxy]propan-2-ol $(270 \mathrm{~m} / z)$; and 1-(4-(prop-1-en-2yl)phenoxy)propan-2-ol $(192 \mathrm{~m} / z)]$. As an example, at $453 \mathrm{~K}$, in the presence of $5 \mathrm{~mol} \%$ of DBU, the quantitative depolymerization of $\mathbf{1}(1.009 \mathrm{~g})$ in 1,2-PD $(10 \mathrm{~mL})$ required a time as long as 9 min (entry 3 , Table 4). Prolonging heating for additional $5 \mathrm{~h}$ caused the complete conversion of $4 \mathrm{a}$ and afforded MHP-BPA (5a' and 5a') and BHP-BPA $\left(\mathbf{6} \mathbf{a}^{\prime}-\mathbf{6} \mathbf{a}^{\prime \prime \prime}\right)$ products, which were isolated (see 2.2 and Figures S4-S7), together with unconverted $\mathrm{BPA}$, by column chromatography (isolated yields: BPA, 38\%; MHP-BPA, 40\%; BHP-BPA, 13\%).

As a whole, the above results show that DBU allows to control the selectivity to cyclic carbonate much better than alkali catalysts, such as $\mathrm{Na}_{2} \mathrm{CO}_{3}$ or $\mathrm{NaOH}$, do. ${ }^{14,15}$ In principle, this aspect of the catalytic activity of DBU can be exploited to improve the selectivity to $\mathbf{4 a}$ by suitably modifying the depolymerization conditions. Accordingly, much better selectivities to $\mathbf{4 a}$ have been achieved by carrying out the depolymerization process in an auxiliary solvent, such as THF (that dissolves 1 easily), applying milder temperature conditions and using the diol in stoichiometric amount versus $\mathrm{PC}\left(\mathrm{mol}_{1,2-\mathrm{PD}} / \mathrm{mol}_{\mathrm{CO}_{3}} \approx 1\right)$.

Figure 4 summarizes the results obtained at different temperatures $(\leq 373 \mathrm{~K})$ when the polymer $(\sim 0.200 \mathrm{~g})$ was

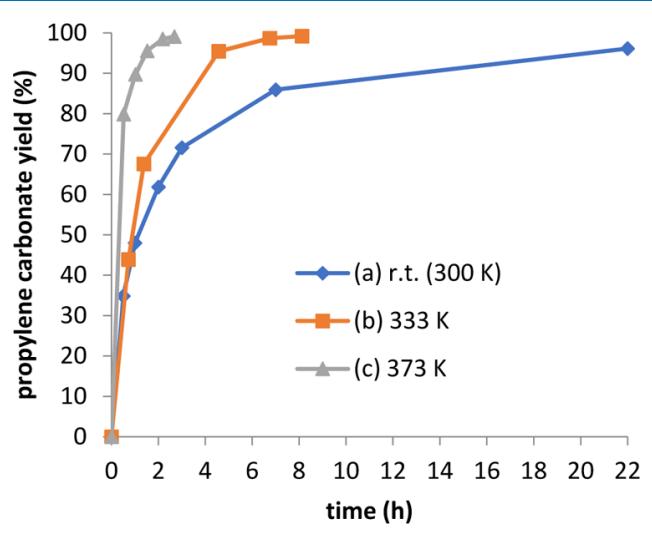

Figure 4. PC $(\sim 0.200 \mathrm{~g})$ depolymerization with 1,2-PD $\left(\mathrm{mol}_{1,2-\mathrm{PD}} /\right.$ $\left.\mathrm{mol}_{\mathrm{CO}_{3}} \approx 1\right)$ in THF $(2 \mathrm{~mL})$ in the presence of DBU $(\sim 10 \mathrm{~mol} \%)$ at different temperatures.

reacted with $3 a$ in THF $(2 \mathrm{~mL})$ in the presence of $10 \mathrm{~mol} \%$ DBU. The progress of the process was monitored by following the formation of $\mathbf{4 a}$ over time. The depolymerization reaction 1 proceeded smoothly even at ambient temperature $(300 \mathrm{~K})$. After 22 h, 4a formed in 96\% yield. Markedly, shorter reaction times were observed at moderately higher temperatures. At $373 \mathrm{~K}$, the depolymerization of $\mathbf{1}$ was quantitative in little more than $2.5 \mathrm{~h}$ with a carbonate yield as high as $99 \%$. Both $4 \mathrm{a}$ and 2 were isolated with high yield $(>90 \%)$ by column chromatography (see 2.3).

The catalyst can be recovered from the reaction mixture as a BPA/DBU adduct, ${ }^{10}$ as described in 2.3.1. Figure $S 8$ shows the ${ }^{1} \mathrm{H}$ NMR spectrum of the adduct. The BPA/DBU molar ratio

Table 4. BPA-Hydroxyalkylation Times by the Glycolysis of Polycarbonate in 1,2-PD $\left(1,2-\mathrm{PD} / \mathrm{PC} \approx 12 \mathrm{~m} / \mathrm{m} ; \mathrm{mol}_{1,2-\mathrm{PD}} /\right.$ $\left.\mathrm{mol}_{\mathrm{CO}_{3}} \approx 3.6\right)$ in the Presence of DBU

\begin{tabular}{|c|c|c|c|c|c|c|c|c|}
\hline entry & 3a $(\mathrm{mL})$ & $\mathrm{PC}(\mathrm{g})$ & $\mathrm{DBU}(\mu \mathrm{L})$ & DBU load ${ }^{a}(\mathrm{~mol} \%)$ & DBU concentration $\mathrm{g}_{\mathrm{DBU}} / \mathrm{mL}_{1,2-\mathrm{PD}}$ & $T(\mathrm{~K})$ & $t_{\text {depolym }}^{b}(\min )$ & $t^{c}(\mathrm{~h})$ \\
\hline 1 & 2 & 0.209 & 12 & 9.9 & 0.006 & 436 & 10 & 4.5 \\
\hline 2 & 2 & 0.199 & 12 & 10.4 & 0.006 & 453 & 5 & 1.5 \\
\hline 3 & 10 & 1.009 & 30 & 5.1 & 0.003 & 453 & 9 & 5 \\
\hline 4 & 2 & 0.204 & 1.2 & 1.0 & 0.0006 & 453 & 21 & 10 \\
\hline
\end{tabular}

${ }^{a}\left(\mathrm{~mol}_{\mathrm{DBU}} / \mathrm{mol}_{\mathrm{CO}_{3}}\right) \times 100 .{ }^{b}$ At that time, the depolymerization of $\mathrm{PC}$ was quantitative (disappearance of the PC pellets). ${ }^{c}$ Overall reaction time (i.e., until the complete conversion of $\mathbf{4 a}$ ). 
was found to be equal to $2.5: 1 .^{10}$ The adduct, once isolated, can be readily reused in a successive run. Even after 5 cycles, both the productivity and selectivity of the process were maintained very high (Figure 5) in accordance with the fact

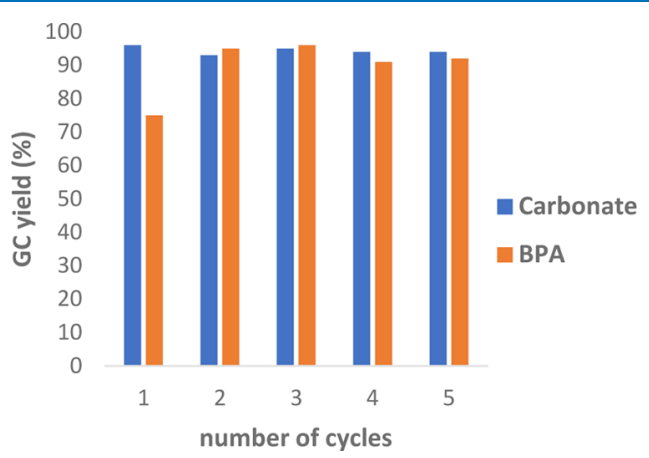

Figure 5. Catalyst recycling: PC, $\sim 0.500$ g; $1,2-\mathrm{PD}, 145 \mu \mathrm{L}$; DBU (used in cycle 1$), 30 \mu \mathrm{L}(\sim 10 \mathrm{~mol} \%)$; THF, $5 \mathrm{~mL} ; 373 \mathrm{~K} ; 2.5 \mathrm{~h}$. After each run, the catalyst was recovered as the BPA/DBU adduct $(2.5: 1 \mathrm{~mol} / \mathrm{mol})$ and reused in the successive cycle. GC yields were determined after the separation of the adduct. This explains why the BPA yield after the first cycle was sensibly lower $(\sim 75 \%)$ than in the successive cycles.

that the catalyst recovered from the last run exhibited spectroscopic features (Figure S9) analogous to those of the catalyst recovered after a single cycle.

3.2. DBU-Promoted Glycerolysis of PC. The study has been extended to an industrially relevant triol such as glycerol (3b). No reaction was observed when a suspension of PC $(0.195 \mathrm{~g})$ in $3 \mathrm{~b}(2.491 \mathrm{~g})$ was stirred for $5 \mathrm{~h}$ at $453 \mathrm{~K}$ in the absence of any catalyst: the polymer was quantitatively recovered by filtration. Therefore, in the absence of any catalyst, the behavior of the polymer in glycerol differs slightly from that displayed in $\mathrm{EG}^{13}$ or 1,2-PD (see 3.1): according to the mechanism proposed by Han, ${ }^{13}$ the very poor reactivity shown by $\mathbf{1}$ in neat glycerol may reflect, most probably, the greater difficulty of the triol molecules in penetrating into the solid particles of the polymer.

However, the addition of a catalytic amount of DBU (10 mol \%) caused a drastic change of reactivity and, under solventless conditions (3b, $2.500 \mathrm{~g})$, promoted the fast depolymerization of $\mathbf{1}(0.200 \mathrm{~g})$. Because of the poor solubility of $\mathbf{1}$ in glycerol, also, in this case, the reaction mixture remained heterogeneous throughout the reaction time, until the disappearance of the pellets. The depolymerization was complete in only $26 \mathrm{~min}$. Nevertheless, under the above conditions, $\mathbf{4 b}$ was obtained in a negligible amount. The GCMS analysis showed the formation of BPA as the major product together with other species, among which we have identified minor amounts of $\mathrm{PhOH}, 4-\mathrm{IPP}$, and BPA hydroxyalkylation products. Use of a less severe temperature (423 K; PC, 0.202 g; glycerol, $2.563 \mathrm{~g}$; DBU, $10 \mathrm{~mol} \%$ ) caused the prolongation of the depolymerization time (disappearance of the PC pellets after $168 \mathrm{~min}$ ) but did not improve the selectivity to $\mathbf{4 b}$. The analysis (FTIR, GC-MS) of the reaction solution, once the depolymerization was complete, showed, in addition to BPA, the formation of minor amounts of glycerol carbonate (no other IR absorptions were noted in the carbonyl region; see also 3.1), which disappeared on prolonging the heating at the working temperature $(423 \mathrm{~K})$ for further $30 \mathrm{~min}$. The monomer $2 \mathrm{can}$ be extracted from the reaction mixture with diethyl ether (GC yield: 90\%) and was isolated (yield: $84 \%$ ) by chromatography on a silica gel column (see Experimental Section). According to the above results, DBU behaves differently from $\mathrm{KOH}$, which under comparable solventless conditions ( $10 \mathrm{~mol} \% ; 423 \mathrm{~K}, 1 \mathrm{~h}$ ) did not show any catalytic activity. ${ }^{18}$

Most likely, the very low selectivity to $4 \mathbf{b}$ was due to the modest stability of glycerol carbonate under the used conditions. The glycerolysis of $\mathbf{1}$ has been, therefore, investigated in THF, at milder temperatures, using a stoichiometric amount of the triol.

Figure 6 shows that, at $373 \mathrm{~K}$, using a catalyst load $\geq 5 \mathrm{~mol}$ $\%$, the quantitative depolymerization of $1(\sim 0.20 \mathrm{~g})$ in THF (2

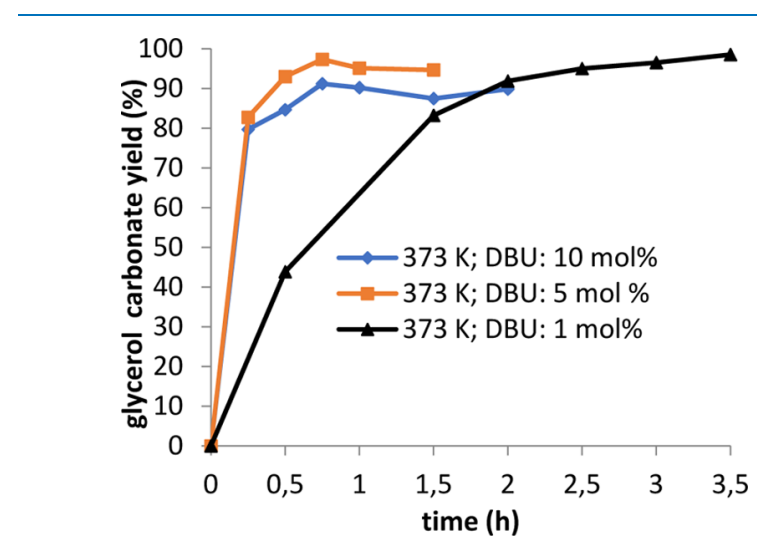

Figure 6. Glycerolysis of 1 (PC $\approx 0.20 \mathrm{~g}$; glycerol: $\sim 60 \mu \mathrm{L}$, $\left.\mathrm{mol}_{\text {glycerol }} / \mathrm{mol}_{\mathrm{CO}_{3}} \approx 1\right)$ catalyzed by DBU, in THF $(2 \mathrm{~mL})$ at $373 \mathrm{~K}$, with different catalyst loads.

$\mathrm{mL})$ required a very short time $(<1 \mathrm{~h})$ : moreover, a sensible increase of selectivity $(90 \rightarrow 96 \%)$ can be observed on decreasing the DBU load. With a catalyst load as low as $1 \mathrm{~mol}$ $\%$, the formation of the cyclic carbonate was even more selective ( $99 \%)$, but the depolymerization process was slower and complete within $3.5 \mathrm{~h}$.

The glycerolysis reaction proceeds quantitatively and selectively, within times acceptable from the applicative point of view, even at temperatures significantly lower than $373 \mathrm{~K}$ (Figure 7). At ambient temperature (293 K), with a $10 \mathrm{~mol} \%$ DBU load, a carbonate yield close to $95 \%$ was achieved within

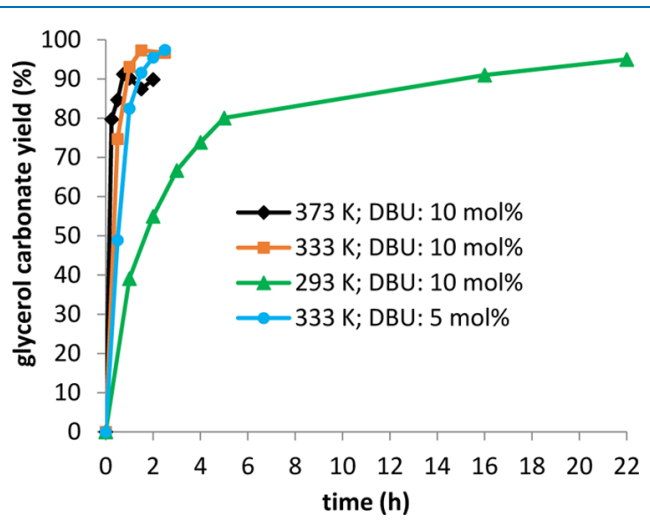

Figure 7. Glycerolysis of 1 (PC $\approx 0.20$ g; glycerol: $\sim 58 \mu \mathrm{L}$, $\left.\mathrm{mol}_{\text {glycerol }} / \mathrm{mol}_{\mathrm{CO}_{3}} \approx 1\right)$ catalyzed by DBU, in THF $(2 \mathrm{~mL})$, at different temperatures. 
$22 \mathrm{~h}$. A comparable yield (97\%) was obtained at $333 \mathrm{~K}$ after only $2.5 \mathrm{~h}$ by using an even lower catalyst load ( $5 \mathrm{~mol} \%$ ).

Also, in this case, the products (BPA and glycerol carbonate) were separated with high yield $(\geq 90 \%)$ by chromatography, as described in 2.5 .

\section{CONCLUSIONS}

In conclusion, under suitable conditions, DBU behaves as an active organocatalyst of PC glycolysis and promotes effectively and selectively the conversion of 1 with $1,2-\mathrm{PD}$ or glycerol to give the monomer (BPA) and the relevant cyclic carbonate $4 \mathbf{a}$ or $\mathbf{4 b}$, respectively.

The depolymerization process can be carried out under solventless conditions (using diol/triol as the reagent and reaction medium) quantitatively with selectivity to carbonate (4a or $4 b$ ), which depends on the used polyol (3a or $3 b$ ), temperature, catalyst load, and reaction time.

Very high selectivities to cyclic carbonate have been obtained by carrying out the depolymerization reaction in a cosolvent, such as THF, wherein the polymer is soluble, and using mild temperature conditions and a stoichiometric amount of polyol.

The depolymerization process does not require any complex apparatus and is simple from the operational point of view. The use of DBU as the catalyst of the process allows to conjugate high productivity with effective catalyst recyclability. Both BPA and the cyclic carbonates $4 \mathbf{a}$ or $\mathbf{4 b}$ have been isolated, at a laboratory scale, in high yield. The above features, while marking positively the described protocols, also provide a suitable starting point for challenging scale up, and, as a whole, make these processes attractive routes to chemical recycling and valorization of the waste polymer.

\section{ASSOCIATED CONTENT}

\section{S Supporting Information}

The Supporting Information is available free of charge on the ACS Publications website at DOI: 10.1021/acsomega.8b01123.

PC (SABIC) characterization (NMR, ATR-FTIR); ${ }^{1} \mathrm{H}$ and ${ }^{13} \mathrm{C}$ NMR of the mixture of MHP-BPA derivatives; ${ }^{1} \mathrm{H}$ and ${ }^{13} \mathrm{C}$ NMR of the mixture of BHP-BPA derivatives; and ${ }^{1} \mathrm{H}$ NMR of the $\mathrm{BPA} / \mathrm{DBU}$ adduct (PDF)

\section{AUTHOR INFORMATION}

\section{Corresponding Author}

*E-mail: eugenio.quaranta@uniba.it. Phone: +390805442093 (E.Q.).

\section{ORCID $\odot$}

Eugenio Quaranta: 0000-0001-7950-9739

Notes

The authors declare no competing financial interest.

\section{ACKNOWLEDGMENTS}

This work was supported by Università degli Studi "Aldo Moro" di Bari (Fondi di Ateneo). LAMIPLAST SRL (Modugno, Bari) is gratefully acknowledged for a generous gift of PC. The authors are indebted to Prof. Francesco Babudri (University of Bari) for molecular weight measurements on PC provided by LAMIPLAST.

\section{REFERENCES}

(1) Handbook of Plastic Recycling; La Mantia, F., Ed.; Rapra Technology: Shrewsbury, U.K., 2002.

(2) Material Recycling-Trends and Perspectives; Achilias, D. S., Ed.; InTech: Rijeka, Croatia, 2012.

(3) Datta, J.; Kopczyńska, P. From polymer waste to potential main industrial products: Actual state of recycling and recovering. Crit. Rev. Environ. Sci. Technol. 2016, 46, 905-946.

(4) Antonakou, E. V.; Achilias, D. S. Recent advances in polycarbonate recycling: A review of degradation methods and their mechanisms. Waste Biomass Valorization 2013, 4, 9-21 and references therein.

(5) Taguchi, M.; Ishikawa, Y.; Kataoka, S.; Naka, T.; Funazukuri, T. $\mathrm{CeO}_{2}$ nanocatalysts for the chemical recycling of polycarbonate. Catal. Commun. 2016, 84, 93-97.

(6) Quaranta, E. Rare Earth metal triflates $\mathrm{M}\left(\mathrm{O}_{3} \mathrm{SCF}_{3}\right)_{3}(\mathrm{M}=\mathrm{Sc}$, $\mathrm{Yb}, \mathrm{La}$ ) as Lewis acid catalysts of depolymerization of poly-(bisphenol A carbonate) via hydrolytic cleavage of carbonate moiety: Catalytic activity of La(O3SCF3)3. Appl. Catal., B 2017, 206, 233-241 and references therein.

(7) Hata, S.; Goto, H.; Yamada, E.; Oku, A. Chemical conversion of polycarbonate to 1,3- dimethyl-2-imidazolidinone (DMI) and bisphenol A. Polymer 2002, 43, 2109-2116.

(8) Hu, L.-C.; Oku, A.; Yamada, E. Alkali-catalyzed methanolysis of polycarbonate. A study on recycling of bisphenol A and dimethyl carbonate. Polymer 1998, 39, 3841-3845.

(9) Liu, F.-S.; Li, Z.; Yu, S.-T.; Cui, X.; Xie, C.-X.; Ge, X.-P. Methanolysis and hydrolysis of polycarbonate under moderate conditions. J. Polym. Environ. 2009, 17, 208-211.

(10) Quaranta, E.; Sgherza, D.; Tartaro, G. Depolymerization of poly(bisphenol A carbonate) under mild conditions by solvent free alcoholysis catalyzed by 1,8-diazabicyclo[5.4.0]undec- 7-ene as a recyclable organocatalyst: a route to chemical recycling of waste polycarbonate. Green Chem. 2017, 19, 5422-5434 and references therein.

(11) North, M.; Pasquale, R.; Young, C. Synthesis of cyclic carbonates from epoxides and $\mathrm{CO}_{2}$. Green Chem. 2010, 12, 15141539.

(12) Fukuoka, S.; Tojo, M.; Hachiya, H.; Aminaka, M.; Hasegawa, K. Green and Sustainable Chemistry in Practice: Development and Industrialization of a Novel Process for Polycarbonate Production from $\mathrm{CO}_{2}$ without Using Phosgene. Polym. J. 2007, 39, 91-114.

(13) Kim, D.; Kim, B.-k.; Cho, Y.; Han, M.; Kim, B.-S. Kinetics of polycarbonate glycolysis in ethylene glycol. Ind. Eng. Chem. Res. 2009, $48,685-691$.

(14) Oku, A.; Tanaka, S.; Hata, S. Chemical conversion of poly(carbonate) to bis(hydroxyethyl) ether of bisphenol A. An approach to the chemical recycling of plastic wastes as monomers. Polymer 2000, 41, 6749-6753.

(15) Lin, C.-H.; Lin, H.-Y.; Liao, W.-Z.; Dai, S. A. Novel chemical recycling of polycarbonate $(\mathrm{PC})$ waste into bis-hydroxyalkyl ethers of bisphenol A for use as PU raw materials. Green Chem. 2007, 9, 38-43.

(16) Nikje, M. M. A. Polymery/Polymers 2011, 56 (5), 381-384.

(17) Rosi, L.; Bartoli, M.; Undri, A.; Frediani, M.; Frediani, P. Synthesis of dianols or BPA through catalytic hydrolysis/glycolysis of waste polycarbonates using microwave heating. J. Mol. Catal. A: Chem. 2015, 408, 278-286.

(18) Hidaka, K.; Iwakawa, Y.; Maoka, T.; Tanimoto, F.; Oku, A. Viable chemical recycling of poly(carbonate) as a phosgene equivalent illustrated by the coproduction of bisphenol A and carbohydrate carbonates. J. Mater. Cycles Waste Manage. 2009, 11, 6-10.

(19) Iannone, F.; Casiello, M.; Monopoli, A.; Cotugno, P.; Sportelli, M. C.; Picca, R. A.; Cioffi, N.; Dell'Anna, M. M.; Nacci, A. Ionic liquids $/ \mathrm{ZnO}$ nanoparticles as recyclable catalyst for polycarbonate depolymerization. J. Mol. Catal. A: Chem. 2017, 426, 107-116.

(20) Carafa, M.; Mesto, E.; Quaranta, E. DBU-promoted nucleophilic activation of carbonic acid diesters. Eur. J. Org. Chem. 2011, 2458-2465. 
(21) Quaranta, E.; Angelini, A.; Carafa, M.; Dibenedetto, A.; Mele, V. Carbonic Acid Diester Activation by Polymer-Bound DBU and Its Relevance to Catalytic N-Carbonylation of N-Heteroaromatics: Direct Evidence for an Elusive N-Carboxy-Substituted Amidinium Cation Intermediate. ACS Catal. 2014, 4, 195-202.

(22) Carafa, M.; Iannone, F.; Mele, V.; Quaranta, E. Solventless selective phosgene-free $\mathrm{N}$ - carbonylation of $\mathrm{N}$-heteroaromatics (pyrrole, indole, carbazole) under mild conditions. Green Chem. 2012, 14, 3377.

(23) Brunelle, D.; Smigelski, P.; Boden, E. Evolution of polycarbonate process technologies. Advances in Polycarbonates; ACS Symposium Series 898; American Chemical Society: Washington, D.C., 2005; pp 8-21.

(24) Perrin, D. D.; Armarego, W. L. F.; Perrin, D. R. Purification of Laboratory Chemicals; Pergamom: Oxford, U.K., 1986.

(25) Schnell, H.; Krimm, H. Formation and Cleavage of Dihydroxydiarylmethane Derivatives. Angew. Chem., Int. Ed. 1963, 2, 373-379. 